\title{
Commentary: Transcatheter aortic valve replacement durability-Wouldn't it be nice if we were older?
}

John Lisko, MD, MPH, and Kendra J. Grubb, MD, MHA, FACC ${ }^{\mathrm{b}}$

From the a Division of Cardiology, Structural Heart and Valve Center, Emory University School of Medicine, Atlanta, Ga; and ${ }^{\mathrm{b}}$ Division of Cardiothoracic Surgery, Structural Heart and Valve Center, Emory University School of Medicine, Atlanta, Ga.

Disclosures: Authors have nothing to disclose with regard to commercial support.

Received for publication June 6, 2019; revisions received July 18, 2019; accepted for publication July 18, 2019; available ahead of print Sept 24, 2019

Address for reprints: Kendra J. Grubb, MD, MHA, FACC, Division of Cardiovascular Surgery, Structural Heart and Valve Center, Emory University School of Medicine, 550 Peachtree St NE, 6th Floor, Atlanta, GA 30308 (E-mail: kjgrubb@emory.edu).

J Thorac Cardiovasc Surg 2020;159:1256-7

$0022-5223 / \$ 36.00$

Copyright (c) 2019 by The American Association for Thoracic Surgery

https://doi.org/10.1016/j.jtcvs.2019.07.105

Recent publication of PARTNER $3^{1}$ and Evolut Low Risk ${ }^{2}$ trials provided further evidence for transcatheter aortic valve replacement (TAVR) as the treatment of severe symptomatic aortic stenosis, demonstrating that TAVR is comparable, possibly superior, to surgical aortic valve replacement (SAVR) in terms of outcomes at 1 year in low-risk patients. Clinicians can confidently counsel patients regarding shortterm outcomes with either TAVR or SAVR; however, they cannot answer a question routinely asked during clinic visits: "Doc, how long will this valve last?"

In this issue of the Journal, Edelman and colleagues ${ }^{3}$ provide a focused review of durability of TAVR and SAVR valves that is based on earlier trials with longer follow-up. Notable findings are as follows:

1. Systematically collected data on the durability of SAVR valves are limited.

2. Surgical data suggest an inverse relationship between patient age and rate of structural valve deterioration (SVD).

3. There does not appear to be accelerated degeneration of TAVR valves implanted in patients considered to be at intermediate, high, or prohibitive risk for SAVR.

4. There are no intermediate (6-10 years) or long-term ( $>10$ years) data regarding SVD in TAVR valves.

This review also raises multiple clinically relevant questions:

1. Can data from a cohort of octogenarian patients (mean age, 79-84 years) be applied to younger, low-risk patients?

2. What is the optimal anticoagulant and antiplatelet strategy in patients undergoing TAVR, and will this effect SVD?

3. What are the risks of TAVR-in-TAVR (valve-in-valve) when the first TAVR fails?

Until systematically collected data are available on long-term follow-up of low-risk patients, clinicians must

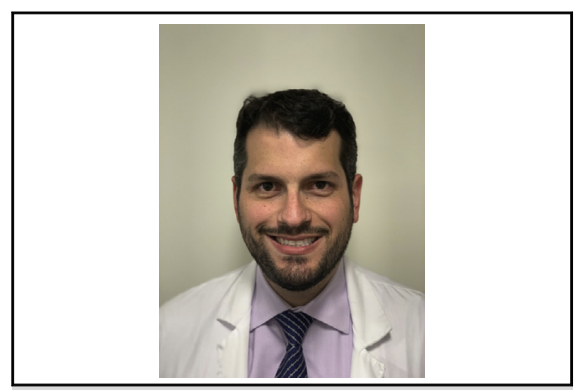

John Lisko, MD, MPH, Structural Heart and Valve Center, Emory University.

\section{Central Message}

TAVR is a promising treatment for severe aortic stenosis in low-risk patients. In discussing options, we must focus not only on 1-year results, but also on the unknowns of limitations and durability.

See Article page 1249 . counsel them on the basis of extrapolations from higher risk trial populations or data collected from registries. These extrapolations are challenging for clinicians to explain and for patients to understand, because many patients are enthusiastic to undergo TAVR to avoid "having my chest cracked."

Further, anticoagulant and antiplatelet agents likely influence durability of all bioprosthetic valves. To date, optimal medication management of patients after TAVR remains unknown. Dedicated studies are needed to determine the optimal strategy to decrease SVD and reintervention.

Without intermediate and long-term durability data, it is difficult to counsel young, low-risk patients on the potential need for another aortic valve replacement. Clinicians need to consider limitations with valve-in-valve TAVR that are not classically associated with redo SAVR: increased gradients and coronary obstruction.

TAVR in young, low-risk patients remains a promising therapy. Until long-term data are available, clinicians must thoroughly counsel patients by acknowledging not only the short-term data but also limitations in our understanding. The low-risk trials highlighted increased rates of patient prosthetic mismatch for balloon-expandable valves and increased permanent pacemaker rates for self-expanding valves, the long-term consequences of which are unknown. Whereas mismatch may affect durability, pacemaker implantation 
affects long-term survival. As with SVD, the consequences of these issues will only be fully understood when long-term data are available. In the era of shared decision making, low-risk patients should be made aware of all treatment options for the management of aortic stenosis, with an emphasis not only on outstanding 1-year results seen in low-risk trials but on the risks and limitations of valve-in-valve TAVR and on the unknowns regarding durability in this patient population.

\section{References}

1. Mack MJ, Leon MB, Thourani VH, Makkar R, Kodali SK, Russo M, et al PARTNER 3 Investigators. Transcatheter aortic-valve replacement with a balloon-expandable valve in low-risk patients. $N$ Engl J Med. 2019;380: 1695-705.

2. Popma JJ, Deeb GM, Yakubov SJ, Mumtaz M, Gada H, O’Hair D, et al; Evolut Low Risk Trial Investigators. Transcatheter aortic-valve replacement with a self-expanding valve in low-risk patients. N Engl J Med. 2019;380: 1706-15.

3. Edelman JJ, Shults C, Thourani VH. Transcatheter aortic valve replacement valve durability: good enough for young, low-risk patients? J Thorac Cardiovasc Surg. 2020;159:1249-55. 